\title{
WiFi Positioning with Propagation-based Calibration
}

\author{
Teemu Pulkkinen* ${ }^{* \dagger}$ \\ *Ekahau Oy \\ Hiilikatu 3 \\ 00180 Helsinki, Finland \\ firstname.lastname@ekahau.com
}

Johannes Verwijnen*†

†Department of Computer Science

University of Helsinki

PO 68, 00014 University of Helsinki, Finland

firstname.lastname@cs.helsinki.fi

\begin{abstract}
Synthetic fingerprint generation using propagation models has been proposed as a cost-effective way to reduce the deployment cost of WiFi positioning systems. Contrary to traditional WiFi positioning systems, which require recording WiFi fingerprints together with ground truth locations, fingerprint generation attempts to automatically populate the radio map using theoretical properties of radio signals. Current solutions for fingerprint generation, however, are extremely complex, requiring complicated modeling of both the signal characteristics and the environment. The present paper contributes by demonstrating that simpler modeling, where only the path-loss exponent is learned from empirical measurements, is sufficient for practical purposes reaching accuracy comparable to carrying out a detailed survey.
\end{abstract}

\section{INTRODUCTION}

WiFi positioning remains the dominant technology for indoor localization for several reasons: (i) WiFi enabled devices are commonplace and widely available; (ii) WiFi positioning can take advantage of existing wireless infrastructures without need for additional infrastructure investments; and (iii) the accuracy of $\mathrm{WiFi}$ positioning is sufficient for most practical indoor use cases. Amongst the myriad of solutions to $\mathrm{WiFi}$ positioning, fingerprinting has been the most successful due to its capability of being able to capture fine-grained variations in the characteristics of the signal environment. However, a significant downside of WiFi fingerprinting is the need for performing a detailed survey by recording large amounts of WiFi measurements together with ground truth locations/labels. These measurements are then used to construct a radio map, which can be used for positioning purposes. As the process of collecting calibration measurements is burdensome and costly, solutions for reducing the calibration effort are essential for boosting the uptake of WiFi positioning systems.

Synthetic fingerprint generation has been proposed as a cost-effective mechanism to reduce the cost of the calibra-

Permission to make digital or hard copies of all or part of this work for personal or classroom use is granted without fee provided that copies are not made or distributed for profit or commercial advantage and that copies bear this notice and the full citation on the first page. To copy otherwise, to republish, to post on servers or to redistribute to lists, requires prior specific permission and/or a fee.

IPSN 2015 Seattle, Washington USA

Copyright 2015 ACM X-XXXXX-XX-X/XX/XX ...\$15.00. tion process $[1,2,3]$. Instead of relying on labeled empirical fingerprints, the idea is to automatically populate the radio map using a theoretical model of radio signal propagation. Current solutions for synthetic fingerprint generation, however, are extremely complex, requiring complicated modeling of both the signal characteristics and the environment. For example, both the ARIADNE [3] and the AROMA [2] systems perform ray tracing based modeling that requires a detailed model of the target environment. Another example is the EZ system [1] which performs complex optimization to find the optimal parameters of a propagation model for the target environment. While these solutions are capable of generating fingerprints that closely match with empirical measurements, it is unclear if such complex modeling indeed is necessary and whether simpler models could be equally effective in terms of positioning accuracy.

The present paper contributes by introducing a simple synthetic fingerprint generation model that considers only the locations of access points and a single propagation parameter (the path-loss exponent). Experiments carried out on measurements collected from a testbed environment demonstrate that, despite using a very simplified model of signal propagation, our approach can reach comparable accuracy to carrying out a real-world survey. Accordingly, our approach provides a cost-effective and complementary solution that can be used as a background model for most areas, and detailed surveying and modeling efforts can be focused on areas where higher accuracy is required.

\section{METHODOLOGY}

Our approach for synthetic fingerprint generation builds on the traditional log-distance path-loss formula, which is expressed as follows:

$$
P L=P L_{0}+10 * \gamma * \log _{10} * \frac{d}{d_{0}}+X_{g} .
$$

Here $P L$ represents the total path loss, $P L_{0}$ the path loss at reference distance $d_{0}, \gamma$ the (environment-specific) pathloss exponent, $d$ the distance from the transmitter, and $X_{g}$ noise, e.g., from multi-path effects. For our purposes, we set $X_{g}=0$ in order to make the approximation entirely deterministic, reproducible and suitable for fitting. For the loss at reference distance, we consider an upper limit value of $P L_{0}=20 \mathrm{dBm}$ at $d_{0}=1 \mathrm{~m}$. The only parameter that we estimate is the path-loss exponent $\gamma$. Note that estimating $\gamma$ also compensates to a large extent the errors in the parameter $P L_{0}$, i.e., loss at reference distance. The exponent used in the experiments was found by fitting propagated 


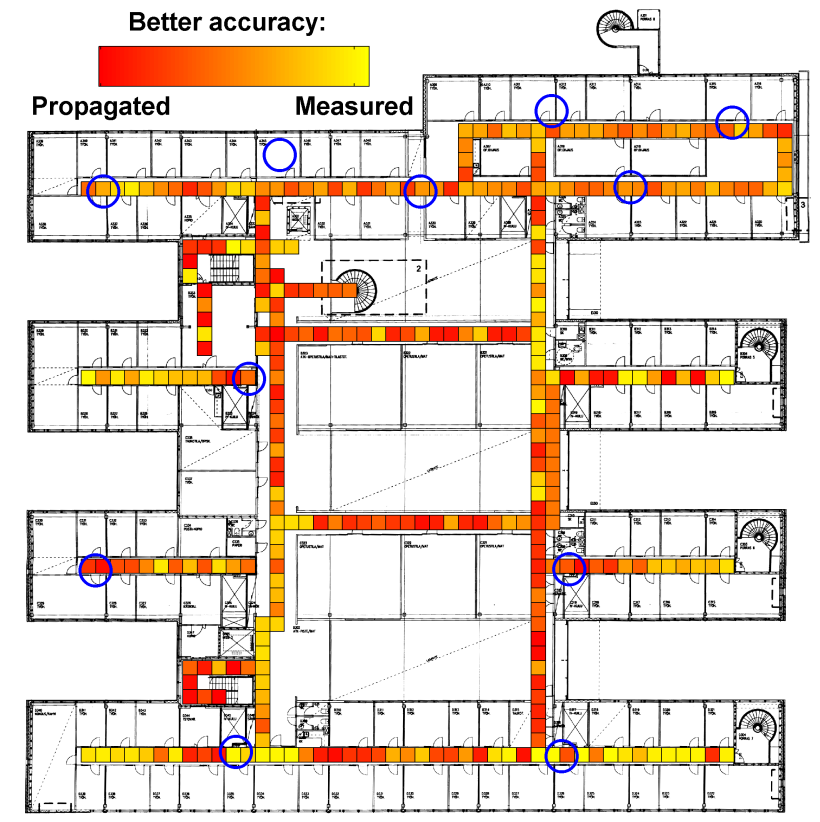

Figure 1: Floorplan of target area with slots depicted as squares and access points as circles.

positioning models against known coordinates and choosing the exponent that lead to the smallest average positioning error. In practice, the model can be learned from a small set of empirical measurements.

\section{EVALUATION}

We evaluate our approach using measurements collected from the third floor of the Exactum building in Helsinki, Finland; see Fig. 1 for a floorplan of the environment. The dataset used for testing was recorded using Ekahau Site Survey $^{1}$ on an ASUS Zenbook laptop. For collecting calibration measurements, two USB-NIC adapters were used simultaneously with a scan rate of $250 \mathrm{~ms}$. For testing we used a single USB adapter and measured the same space covered during calibration, scanning at about $1 \mathrm{~Hz}$. After calibration, the model contained parameters for (on average) 5 access points per slot based on approximately 170 scans per slot. Test data was collated on a per-second basis, meaning we emulated receiving one WiFi fingerprint per second, which is traditionally the expected positioning rate. At this rate we ended up with 830 test fingerprints.

We consider a probabilistic WiFi positioning system, originally introduced by Roos et al. [4]. We assume the system is trained on top of a grid-based model that divides the floorplan of the target environment into discrete areas or slots. As a baseline for our evaluation, we consider an empirical approach which uses the mean and standard deviation within a cell as the parameters of the probabilistic model. We compare this model against a model where the fingerprints have been synthetically generated using our approach. With both models, positioning was performed by calculating the Gaussian pdf for each access point based on measurements collected separately during the testing phase, after which the

\footnotetext{
${ }^{1}$ Ekahau Site Survey (ESS) http://www.ekahau.com/ wifidesign/ekahau-site-survey
}

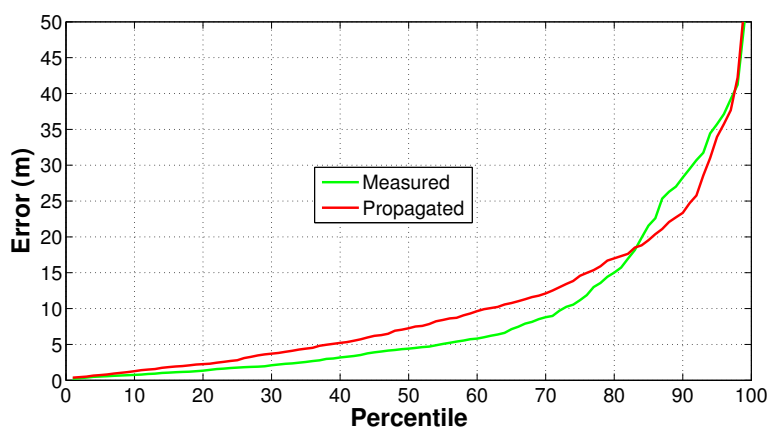

Figure 2: Percentile of Euclidean error in meters for the two approaches.

total mass for each slot was estimated as the product over these. The final position estimation is then the slot with the highest mass.

The results of our evaluation are shown in Fig. 2. As can be observed from the results, the positioning accuracy of our approach is reasonable (i.e., room level) overall, and even manages to decrease positioning errors at higher percentiles (i.e., 80-percentile and above) compared to the empirical model. Accordingly, our simple approach complements previous works by providing a cost-effective solution for generating the radio map. Our solution can also be adopted as a kind of "default" radio map that is sufficient for most practical use cases. As better accuracy is typically required only within certain areas, the calibration efforts can then be focused more on these areas instead of attempting to cover the entire environment densely. Synthetically generated radio maps also provide a viable solution for environments that are too cumbersome to survey manually. Finally, propagation model based fingerprints are also well-suited for remote "debugging", enabling adjustments to be tested remotely and to update the system parameters without involving end-users.

\section{Acknowledgments}

This work was supported by the Finnish Funding Agency for Technology and Innovation (project D2I).

\section{REFERENCES}

[1] K. Chintalapudi, A. P. Iyer, and V. N. Padmanabhan. Indoor localization without the pain. In Proceedings of the 16th Annual International Conference on Mobile Computing and Networking (MobiCom), 2010.

[2] A. Eleryan, M. Elsabagh, and M. Youssef. Synthetic generation of radio maps for device-free passive localization. In Proceedings of the Global Communications Conference (GLOBECOM), 2011.

[3] Y. Ji, S. Biaz, S. Pandey, and P. Agrawal. ARIADNE: a dynamic indoor signal map construction and localization system. In Proceedings of the 4 th International Conference on Mobile Systems, Applications, and Services (MobiSys), 2006.

[4] T. Roos, P. Myllymäki, H. Tirri, P. Misikangas, and J. Sievänen. A probabilistic approach to WLAN user location estimation. International Journal of Wireless Information Networks, 9(3):155-164, 2002. 


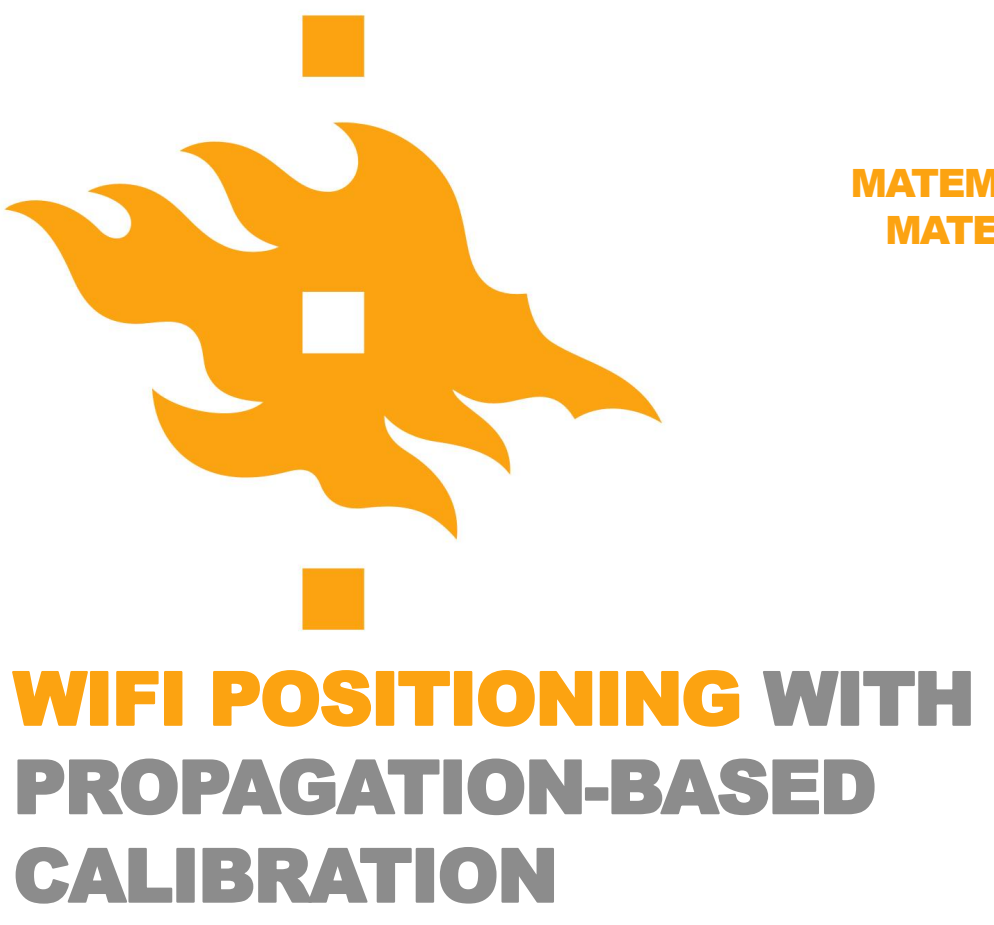

HELSINGIN YLIOPISTO HELSINGFORS UNIVERSITET UNIVERSITY OF HELSINKI MATEMAATTIS-LUONNONTIETEEㄴㄴNNEN TIEDEKUNTA MATEMATISK-NATURVETENSKAPLIGA FAKULTETEN

FACULTY OF SCIENCE

\section{ABSTRACT}

WiFi fingerprinting is a popular technique for indoor positioning, but the cost of deploymen might make some environments unfeasible.

We show that generating fingerprints using a simple propagated model can achieve surprisingly good accuracy, with only one parameter to optimize for.

\section{METHODOLOGY}

The third floor of the Exactum building in Kumpula, Helsinki was divided into "slots", which were initialized to contain the Gaussian parameters of the access points heard within them

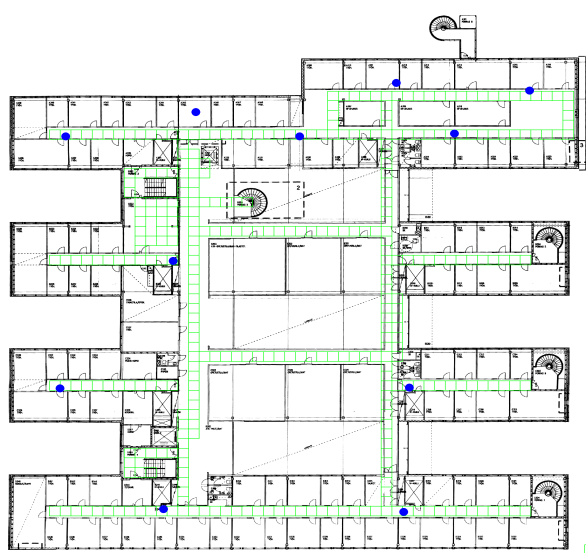

$3^{\text {rd }}$ floor of Exactum, with slot layout and access points depicted.

In a separate model, the slots were populated using the standard path-loss formula:

$P L=P L_{0}+10 * \gamma * \log _{10} * \frac{d}{d_{0}}+X_{g}$

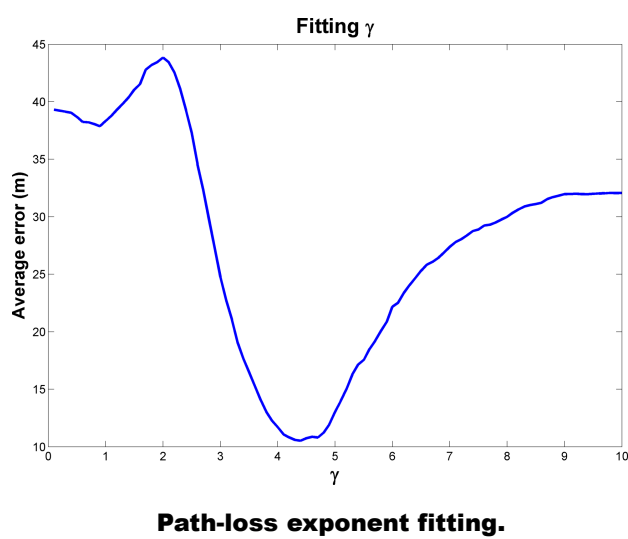

The path-loss exponent was found by fitting the propagation model w.r.t. the known ground-truth.

The models were then compared in terms of positioning accuracy

\section{RESULTS}

The propagated model performed well overall, and even better than the measured model in some worst-case locations.

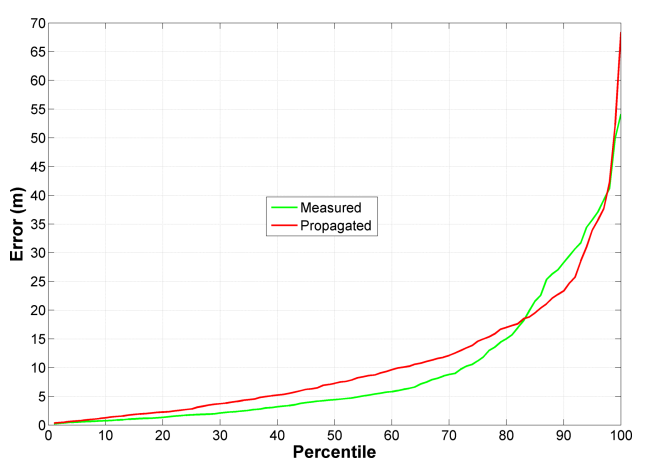

Propagated model accuracy is good compared to measured model.

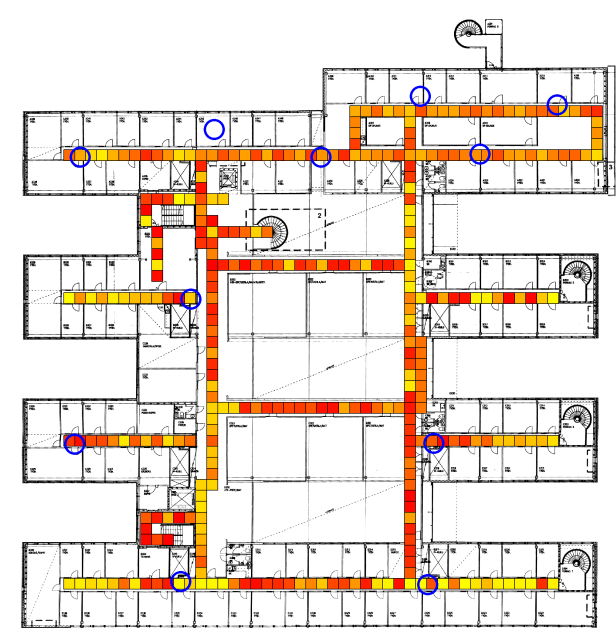

Differences in accuracy as a heatmap (red = propagated, yellow $=$ measured).

As expected, the propagated model does better the less infrastructure (and distance) is between the access point and estimated location. The measured model seems to do better in confined locations, such as the end of hallways.

\section{-UTURE WORK}

Fitting the path-loss exponent in a partially unsupervised way based on limited sampling, and deployment in more complex environments with more access points.

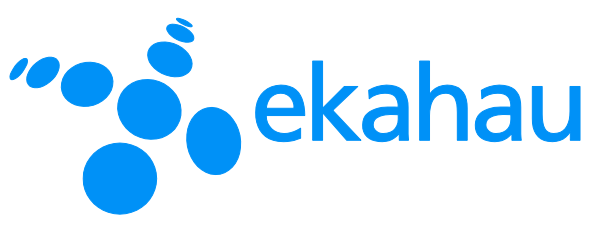

